\title{
MOTOR-BASED CONTROL OF MANIPULATORS WITH FLEXIBLE JOINTS AND LINKS
}

\author{
King Yuan \\ Department of Mechanical Engineering \\ National Taiwan University \\ Taipei, Taiwan 10764, R.O.C.
}

\author{
Lih-Chang Lin \\ Department of Mechanical Engineering \\ National Chung Hsing University \\ Taichung, Taiwan 40227 , R.O.C.
}

\begin{abstract}
A motor-based decoupling and partial (input-output) linearization approach to the control of multi-link robots with joint and link flexibilities is studied in this paper. The control strategy consists of two parts: nominal tracking control and perturbed stabilization control. The nominal tracking control derived by the differential geometric structure algorithm is an input-output decoupling and partial linearization feedback law capable of precise motor-based trajectory tracking, but the zero dynamics of the unobservable nonlinear elastic subsystem remains unstable. In order to actively suppress the elastic vibrations, it is necessary to sacrifice the perfect motor-based tracking capability somewhat by introducing a perturbation control in the vincinity of a desired trajectory. The perturbed stabilization control synthesized by combined LQR and servocompensator is used to achieve active damping of elastic vibration and robust tracking of motor dynamics. To offset the tracking errors of the end effector caused by joint and link deflections due to gravity, the quasi-static deflections can be taken into account in the trajectory planning and LQ regulator. A two-link arm is tested via simulation.
\end{abstract}

\section{INTRODUCTION}

The flexibility of a lightweight robot arm may consist of distributed link flexibility and/or lumped joint elasticity. Because of the ex treme complexity of the dynamic equations of motion for multi-link manipulators with both joint and link flexibilities, numerous literature on the control of flexible manipulators have studied arms with either joint elasticity or link flexibility, and have treated only special configurations or single link examples. Some attempt has been made to develop control strategies for manipulators having two elastic links but with rigid joints [1-11]. Only few work has addressed the control problems of two link/ two joint systems where both structural and joint flexibility exist [12-14]. Recent studies on the dynamic simulations of onelink and two-link robot manipulator with both structural and joint flexibility $[15,16]$ have revealed that, among other things, there is no rigid mode for the flexible joint system. As such, both structural and joint flexibility must be considered in the analysis and control of such systems. However, it is the motor that drives elastic transmission system and the motor dynamics is itself linear. Therefore, a simple and perhaps very effective approach to the end effector trajectory tracking is to design a feedback control law that decouples motor dynamics and stabilizes the elastic vibrations simultaneously. The decoupled motor dynamics are, of course, be designed to achieve precise tracking of the end effector. In this paper, we will show that this goal can be approximately accomplished.

Our approach is as follows. By applying the differential geometric structure algorithm [17], a multi-link flexible dynamic system can be decomposed, via a state-feedback, into two subsystems: a decoupled linear time-invariant motor dynamics relating each motor torque input and its associated motor angular positional output, and an unobservable nonlinear flexure dynamics which do not affect the positional output of each motor. The decoupled linear motor dynamics now admit an exact motor-based tracking of all feasible smooth trajectories if there is matching in initial conditions. However, any statefeedback which is used to achieve a perfect motor-based tracking with a desired linear dynamics, is not able to stabilize the end effector vibrations unless some passive damping is present. This observation is closely related to the unstable zero dynamics as mentioned in [18]. Therefore, in order to stabilize the unobservable nonlinear flexure dynamics, it is imperative that the linear motor dynamics subsystem and nonlinear flexure subsystem are recoupled. In other words, in order to actively suppress the elastic vibrations, it is necessary to sacrifice the perfect motorbased tracking capability somewhat by introducing a perturbation control in the vincinity of a desired trajectory. The perturbed stabilization control synthesized by combined LQR and servocompensator can be used to achieve active damping of elastic vibrations and robust tracking of motor motion. Although there is a tradeoff between the stabilization of elastic vibrations and the motor tracking accuracy, a fairly accurate tracking is still possible with a properly designed perturbation control. To offset the tracking errors of the end effector caused by joint and link deflections due to gravity, the quasi-static deflections can be taken into account in the trajectory planning and LQ regulation. The proposed control strategy will be shown to be effective through computer simulation for a two-link arm with both joint and link flexibility.

A distinct feature of our approach is that the feedback control law is linear and simple. This is due to the presence of linear joint elasticity. If the joints are rigid, a more complicated nonlinear feedback control law will be resulted $[9,19]$.

\section{DYNAMIC MODEL}

We now consider an n-link flexible manipulator with revolute flexible joints modeled by linear torsional springs with known stiffnesses $k_{i}$ 's as shown in Fig. 1. The payload is modeled as a rigid body with mass and moments of interia. Under the assumptions of small link deflections and negligible twist deformations, the flexible motions of the links from their rigid body 
positions can be expressed through modal analysis based on the Euler-Bernoulli beam theory as

$$
\begin{aligned}
& \delta_{x, i}(\eta, t)=\underset{j=1}{N_{i}} p_{i j}(t) \Phi_{i j}(\eta) \\
& \delta_{y, i}(\eta, t)=\sum_{j=1}^{N_{i}} q_{i j}(t) \Psi_{i j}(\eta) \\
& \delta_{z, i}(\eta, t)=\sum_{j=1}^{N_{i}} r_{i j}(t) \Omega_{i j}(\eta),
\end{aligned}
$$

where $\Phi_{\mathrm{ij}}, \Psi_{\mathrm{ij}}$ and $\Omega_{\mathrm{ij}}$ are the jth shape functions of the deflections of the ith link along the $x, y, z$ axis, respectively; and $\mathrm{p}_{\mathrm{ij}}, \mathrm{q}_{\mathrm{ij}}$ and $\mathrm{r}_{\mathrm{ij}}$ are the corresponding generalized coordinates, respectively; $N_{i}$ is the number of modes used to describe the deflections of link $i$; and $n$ is the longitudinal coordinate that may be $\mathrm{x}$, or $\mathrm{y}$, or $\mathrm{z}$, depending on the link coordinate systems constructed for describing the spatial motions.

The kinetic and potential energy expressions for a flexible robot can then be derived. A general explicit closed form Lagrange-Euler/assumed modes dynamics formulation has been proposed in [20] via the Lagrangian approach. The full nonlinear equations of motion for any specific flexible arm can be obtained by computing only the dynamic coefficients through the use of computer algebra such as MACSYMA, and can be put in the following form

$$
\underline{\mathrm{D}}(\underline{\mathrm{q}}) \underline{\ddot{q}}+\underline{\mathrm{C}}(\underline{\mathrm{q}}, \underline{\dot{q}})+\underline{\mathrm{e}}(\underline{\mathrm{q}})=\underline{\mathrm{B}} \underline{\underline{q}}
$$

where $\underline{q}=\left[\underline{q}_{m}^{T}, q_{1}^{T}, q_{2}^{T}\right]^{T}$ is the $N \times 1$ vector of all generalized coordinates of the flexible robot; $\underline{q}_{m}=\left[q_{m 1}, \ldots, q_{m n}\right]^{T}$ is the vector of motor displacements; $q_{1}=\left[q_{1}, \ldots, q_{n}\right]^{T}$ is the vector of effective link displacements; $q_{2}=\left[p_{11}, \ldots \ldots\right.$, $\mathrm{p}_{1 \mathrm{~N}_{1}}, \mathrm{q}_{11}, \ldots \ldots \mathrm{q}_{1 \mathrm{~N}_{1}}, \mathrm{r}_{11}, \ldots, \mathrm{r}_{1 \mathrm{~N}_{1}}, \mathrm{p}_{21}, \ldots \ldots, \mathrm{r}_{2 \mathrm{~N}_{2}}$, $\ldots \ldots, p_{n 1}, \ldots \ldots, p_{n N_{n}}, q_{n 1}, \ldots \ldots, q_{n N_{n}}, r_{n 1}, \ldots \ldots$ $\left.r_{n N}\right]^{T}$ is the vector of all generalized coordinates of link deformations; $\underline{D}(\underline{\mathrm{q}})=$ Block diag. $\left[\mathrm{D}_{\mathrm{j}}, \underline{D}_{\ell}\left(\underline{\mathrm{q}}_{1}, \underline{\mathrm{q}_{2}}\right)\right]$ is the $\mathrm{N} \times \mathrm{N}$ symmetric positive definite inertia matrix; $\underline{D}_{j}=\operatorname{diag} .\left[J_{r l}, \ldots \ldots\right.$, $\left.\mathrm{J}_{\mathrm{Yn}}\right]$ is the diagonal joint inertia matrix, $\mathrm{J}_{\mathrm{ri}}=\mathrm{J}_{\mathrm{mi}}+\mathrm{J}_{\mathrm{fi}} / \mathrm{n}_{\mathrm{i}}^{2}$ is the inertia of the ith joint reflected on the motor side; $\underline{\mathrm{D}}\left(\underline{q}_{1}, \underline{q}_{2}\right)=$ the $(\mathrm{N}-\mathrm{n}) \times(\mathrm{N}-\mathrm{n})$ symmetric positive definite inertia matrix of the corresponding flexible robot with only link flexibility; $\underline{C}(\underline{q}, \dot{q})=\left[0_{n \times 1}^{\mathrm{T}}, \underline{C}_{\ell}^{\mathrm{T}}(\underline{\mathrm{q}}, \underline{\dot{\mathrm{q}}})\right]^{\mathrm{T}} ; \underline{\mathrm{C}}_{\ell}(\underline{\mathrm{q}}, \dot{\mathrm{q}})=$ the $(\mathrm{N}-\mathrm{n}) \times 1$ vector that contains the nonlinear coupling terms representing the Coriolis, centripetal and gravitational forces/torques of the corresponding flexible robot with only link flexibility; $\mathrm{e}(\mathrm{q})=$ $\left[\underline{e}_{1}^{T}\left(\underline{q}_{m}, \underline{q}_{1}\right), \underline{e}_{2}^{T}\left(\underline{q}_{m}, \underline{q}_{1}\right),\left(\underline{K}_{l} \underline{q}_{2}\right)^{T}\right]^{T} ; \underline{e}_{1}\left(\underline{q}_{m}, \underline{q}_{1}\right)=\left[\left(\bar{k}_{1} / n_{1}\right)\right.$ $\left.\left(q_{m 1} / n_{1}-q_{1}\right), \ldots \ldots,\left(k_{n} / n_{n}\right)\left(q_{m n} / n_{n}-q_{n}\right)\right]^{T}$ is the $n \times 1$ elastic forces/torques reflected on the motor side; $\mathrm{e}_{2}\left(\mathrm{q}_{\mathrm{m}}, \mathrm{q}_{1}\right)=$ $\left[k_{1}\left(q_{1}-q_{m 1} / n_{1}\right), \ldots \ldots, k_{n}\left(q_{n}-q_{m n} / n_{n}\right)\right]^{T}$ is the $n \times 1$ elastic forces/torques reflected on the link side; $K_{2}=$ the $(N-2 n)$ $X(N-2 n)$ stiffness matrix of the $n$-links, depending on the number of modes and modal shape functions used; $\underline{B}=U_{n \times n}$, $\left.\underline{0}_{\mathrm{n} \times(\mathrm{N}-\mathrm{n})}\right]^{\mathrm{T}} ; \tau=\left[\tau_{\mathrm{m} 1}, \ldots \ldots \tau_{\mathrm{mn}}\right]^{\mathrm{T}}$ is the actuator torque vector.

\section{STATIC STATE-FEEDBACK VIA STRUCTURE ALGORITHM}

3.I Formulation of State-Space Dynamics Model

If we choose the state vector as $\underline{x}=\left(\underline{x}_{1}^{T}, \underline{x}_{2}^{T}\right)^{T}=\left(q_{1}-q_{m 1} /\right.$ $\mathrm{n}_{1}, \ldots, \mathrm{q}_{\mathrm{n}}-\mathrm{q}_{\mathrm{mn}} / \mathrm{n}_{\mathrm{n}}, \underline{\mathrm{q}}_{2}^{\mathrm{T}}, \underline{\mathrm{q}}_{\mathrm{m}}^{\mathrm{T}}, \dot{\mathrm{q}}_{1}-\dot{\mathrm{q}}_{\mathrm{m} 1} / \mathrm{n}_{1}, \ldots, \dot{\mathrm{q}}_{\mathrm{n}}-\dot{\mathrm{q}}_{\mathrm{mn}} / \mathrm{n}_{\mathrm{n}}$,
$\left.\dot{\mathrm{q}}_{2}^{\mathrm{T}}, \dot{\mathrm{q}}_{\mathrm{m}}^{\mathrm{T}}\right)^{\mathrm{T}}$, and select the motor displacements divided by their reduction ratios $\left(n_{i}\right.$ 's), respectively, as the output variables, then the state-space dynamic model can be written as

$$
\begin{aligned}
& \underline{\dot{x}}=\underline{f}(\underline{x})+\underline{g}(\underline{x}) \tau \\
& \underline{y}=\underline{h}(\underline{x})=\left(q_{m 1} / n_{1}, q_{m 2} / n_{2}, \ldots, q_{m n} / n_{n}\right)^{T}
\end{aligned}
$$

where

$$
\begin{aligned}
& \underline{\mathrm{f}}(\underline{\mathrm{x}})=\left(\begin{array}{c}
\underline{\mathrm{x}}_{2} \\
-\underline{\mathrm{D}}^{\prime-1}\left(\underline{\mathrm{C}}^{\prime}+\underline{\mathrm{e}}^{\prime}\right)
\end{array}\right)+\left(\begin{array}{c}
\underline{\mathrm{o}}_{\mathrm{N} \times 1} \\
-\left(\underline{\left.\mathrm{N} \underline{D}_{j}\right)^{-1} \underline{\mathrm{e}}_{1}\left(\underline{\mathrm{q}}_{\mathrm{m}}, \underline{\mathrm{q}}_{1}\right)}\right. \\
--\underline{\mathrm{o}}_{(\mathrm{N}-\mathrm{n}) \times 1}
\end{array}\right) ; \\
& \underline{g}(x)=\left(\begin{array}{c}
\underline{0}_{N} \times n \\
--\left(N_{-} \underline{D}_{j}\right)^{-1} \\
\hdashline \underline{0}_{(N-2 n) \times n} \\
\hdashline \underline{D}_{j}^{-1}
\end{array}\right) ; \quad \underline{D}^{\prime}=\left(\begin{array}{c|c}
\underline{D}_{l} & \underline{0} \\
\hdashline \underline{0} & \underline{D}_{j}
\end{array}\right)
\end{aligned}
$$

is the symmetric positive definite inertia matrix; $\underline{C}^{\prime}=\left[C_{Q}^{T}(\underline{q}, \dot{q})\right.$, $\left.0^{\mathrm{T}}\right]^{\mathrm{T}} ; \mathrm{e}^{\prime}=\left[\mathrm{e}^{\mathrm{T}}\left(\mathrm{q}_{\mathrm{m}}, \mathrm{q}_{1}\right),\left(\mathrm{K}_{\mathrm{l}} \mathrm{q}_{2}\right)^{\mathrm{T}}, \mathrm{e}^{\mathrm{T}}\left(\mathrm{q}_{\mathrm{m}}, \mathrm{q}_{1}\right)\right]^{\mathrm{T}} ; \hat{\mathrm{N}}=$ diag. $\left[n_{1}, \ldots \ldots, n_{n}\right]$ is then $n \times n$ diagonal reduction ratio matrix.

\subsection{Construction of Input-Output Decoupling and Linearizing}

Feedback

As the dynamic equations (3) are highly nonlinear, coupled and complex, the differential geometric approach to the construction of an input-output decoupling and linearizing feedback control suitable for the motor-based tracking appears to be attractive. In fact, an elegant recursive procedure known as structure algorithm [17] naturally leads to such linearizing feedback law as

$$
\underline{I}(\underline{x})=\underline{\alpha}(\underline{x})+\underline{\beta}(\underline{x}) \underline{u}
$$

where $\mathrm{u}$ is the new $\mathrm{n} \times 1$ input vector. The algorithm will terminate at step 2, and the feedback functions $\alpha(x)$ and $\beta(x)$ can then be constructed by the following two equations:

$$
\left[\mathrm{L}_{\underline{g}} \underline{\Gamma}(\underline{\mathrm{x}})\right] \underline{\alpha}(\underline{\mathrm{x}})=-\mathrm{L}_{\underline{\mathrm{f}}} \underline{\Gamma}(\underline{\mathrm{x}}) ; \quad\left[\mathrm{L}_{\underline{g}} \underline{\Gamma}(\underline{\mathrm{x}})\right] \underline{\beta}(\underline{\mathrm{x}})=\omega_{0}^{2} \underline{\mathrm{I}}_{\mathrm{n} \times \mathrm{n}}
$$

where

$$
\begin{aligned}
& \underline{\Gamma}(\underline{x})=L_{\underline{f}} \underline{h}(\underline{x}) ; \quad L_{\underline{g}} \underline{\Gamma}(\underline{x})=L_{\underline{g}} L_{\underline{\underline{ }}} \underline{h}(\underline{x})=\left(\underline{N}_{\underline{j}}\right)^{-1} \triangleq \underline{G}_{a} \\
& L_{\underline{f}} \Gamma(\underline{x})=L_{\underline{f}}^{2} \underline{h}(\underline{x})=\left(\underline{N}^{2} \underline{D}_{j}\right)^{-1}\left(k_{1} x_{1}, k_{2} x_{2} \ldots k_{n} x_{n}\right)^{T} \triangleq \underline{G}_{f},
\end{aligned}
$$

$\omega_{0}$ is the selected natural frequency for the linear input-output pairs; $L_{\mathrm{f}} \underline{\mathrm{h}}$ is the Lie derivative of $\underline{h}$ along $\mathrm{f}$; and similarly for others. Thus we can obtain

$$
\begin{aligned}
& \underline{\alpha}(\underline{x})=-\underline{G}_{a}^{-1} G_{f}=-\underline{N}^{-1}\left[k_{1} x_{1}, \ldots, k_{n} x_{n}\right]^{T} \\
& \underline{\beta}(\underline{x})=\omega_{0}^{2} \underline{G}_{a}^{-1}=\omega_{0}^{2} \underline{N} \underline{D}_{j}
\end{aligned}
$$

Here $\alpha(x)$ means that the elastic torques driving the links can be computed or measured for use as the feedback function, and 
$\underline{\beta}(\underline{x})$ is only a constant matrix.

\subsection{Internal Structure of the Closed-doop System}

Substitute the state-feedback law into (3), and let $\underline{z}=$ $\left[(\underline{h}(\underline{x}))^{T}\left(L_{\underline{f}} h(\underline{x})\right)^{T}\right]^{T}=\left(q_{m 1} / n_{1}, q_{m 2} / n_{2}, \ldots, q_{m n} / n_{n}, \dot{q}_{m 1} /\right.$ $\left.n_{1}, \ldots, \dot{q}_{m n} / n_{n}\right)^{T}$, then the internal structure of the closed-loop system composed of two parts can be obtained as

$$
\begin{array}{ll}
\underline{\dot{z}}=\underline{A}_{z}^{\prime} \underline{z}+\omega_{0}^{2} \underline{B}_{z}^{\prime} \underline{\mathrm{u}} & \text { linear subsystem } \\
\underline{\dot{n}}=\underline{\mathrm{f}}_{\eta}(\underline{\mathrm{z}}, \underline{\eta})+\underline{\mathrm{g}}_{\eta}(\underline{\mathrm{z}}, \underline{\eta}) \underline{\mathrm{u}} & \text { nonlinear subsystem } \\
\underline{\mathrm{y}}=[\underline{\mathrm{I}} \underline{0}] \underline{\mathrm{z}} &
\end{array}
$$

where

$$
\underline{\mathbf{A}}_{\mathrm{z}}^{\prime}=\left(\begin{array}{c|c}
\underline{0} & \underline{\mathbf{I}} \\
\hline \underline{0} & \underline{0}
\end{array}\right) \quad ; \quad \underline{\mathbf{B}}_{\mathrm{z}}^{\prime}=\left(\begin{array}{c}
\underline{0} \\
\hdashline \underline{\mathbf{I}}
\end{array}\right) \quad ;
$$

$\eta=\left(q_{1}-q_{m 1} / n_{1}, \ldots, q_{n}-q_{m n} / n_{n}, q_{2}^{T}, \dot{q}_{1}-\dot{q}_{m 1} / n_{1}, \ldots\right.$ $\left.\dot{\mathrm{q}}_{\mathrm{n}}-\dot{\mathrm{q}}_{\mathrm{mn}} / \mathrm{n}_{\mathrm{n}}, \dot{\mathrm{q}}_{2}^{\mathrm{T}}\right)^{\mathrm{T}}$ is the vector of all elastic generalized coordinates; $y$ is the output vector; $I$ is the $n X n$ identity matrix; $\underline{f}_{\eta}=\underline{L}[\underline{f}(\underline{x})+\underline{g}(\underline{x}) \underline{\alpha}(\underline{x})] ; \underline{g}_{\eta}=\underline{L}[\underline{g}(\underline{x}) \underline{\beta}(\underline{x})]$; and

$$
\underline{L}=\left(\begin{array}{llll}
\underline{\mathbf{I}}_{(\mathrm{N}-\mathrm{n})} & \underline{0}_{(\mathrm{N}-\mathrm{n}) \times \mathrm{n}} & \underline{0} & \underline{0} \\
\underline{0} & \underline{0} & \underline{\mathbf{I}}_{(\mathrm{N}-\mathrm{n})} & \underline{0}_{(\mathrm{N}-\mathrm{n}) \times \mathrm{n}}
\end{array}\right)
$$

\subsection{Pole Placement for Desired Spectral Characteristics of the} Linear Subsystem

The linear part of the closed-loop system is a decoupled n-channel second-order system with eigenvalues all at origin. Its poles can be arbitrarily placed by constant state feedback. To have appropriate rise time and nearly no overshoot, the desired poles can be placed at those of the Bessel transfer function [21], i.e., $s_{1,2}=-0.8660 \omega_{0} \pm j 0.5000 \omega_{0}$, where $\omega_{0}$ determines the rise time. And the feedback law $\underline{K}$ is

$$
\underline{\mathrm{K}}=\left[-\underline{\mathrm{I}}_{\mathrm{n} \times \mathrm{n}}-\left(1.732 / \omega_{0}\right) \underline{\mathrm{I}}_{\mathrm{n} \times \mathrm{n}}\right]
$$

Thus, the new overall feedback functions are

$$
\underline{\alpha}^{\prime}(\underline{x})=\underline{\alpha}(\underline{x})+\underline{\beta}(\underline{x}) \underline{\mathbf{K}} \underline{z} ; \quad \underline{\beta^{\prime}}(\underline{x})=\underline{\beta}(\underline{x})
$$

and the static state-feedback law is

$$
\underline{\tau}=\underline{\alpha}^{\prime}(\underline{x})+\underline{\beta}^{\prime}(\underline{x}) \underline{v}
$$

where $\underline{v}$ is the new $\mathrm{n} \times 1$ input vector. Then the linear part of the closed-loop system is

$$
\underline{\dot{z}}=\underline{A}_{z} \underline{z}+\omega_{0}^{2} \underline{B}_{z} \underline{v} ; \quad \underline{y}=\left[\begin{array}{ll}
\underline{1} & \underline{0}
\end{array}\right] \underline{z}
$$

where

$$
\underline{A}_{2}=\left(\begin{array}{c|c}
\underline{O}_{\mathrm{n} \times \mathrm{n}} & \underline{I} \\
\hline-\omega_{0}^{2} \underline{I} & -1.732 \omega_{0} \underline{I}_{\mathrm{n} \times \mathrm{n}}
\end{array}\right)
$$

$$
\underline{B}_{z}=\left(\frac{\underline{0}_{n \times n}}{\omega_{0}^{2} \underline{I}_{n \times n}}\right)
$$

and the nonlinear part is

$$
\dot{\eta}=\underline{f}_{\eta}(\mathbf{z}, \underline{\eta})+\underline{g}_{\eta}(\underline{z}, \underline{\eta}) \underline{\mathbf{K}} \mathbf{z}+\underline{g}_{\eta}(\underline{\mathbf{z}}, \underline{\eta}) \underline{v}
$$

\subsection{Command Generation for Output Tracking}

If $\underline{h}_{d} \in R^{n}$ is the desired outputs, and the nominal command inputs $\underline{v}_{\mathrm{d}}$ are selected as

$$
\underline{v}_{d}=\left(1 / \omega_{0}^{2}\right) \ddot{\underline{h}}_{d}+\left(1.732 / \omega_{0}\right) \underline{\underline{h}}_{d}+\underline{h}_{d}
$$

then we can obtain the error equations of the system for output tracking as

$$
\ddot{\widetilde{h}}_{i}+1.732 \omega_{0} \dot{\tilde{h}}_{i}+\omega_{0}^{2} \widetilde{h}_{i}=0, \quad i=1,2, \ldots, n
$$

where $\tilde{h}_{i}=h_{i}-h_{d, i}$ and $h=\left[h_{1}, \ldots, h_{n}\right]^{T}$ is the actual output $y$. Note that if the initial conditions of $\underline{h}$ and $\underline{h}_{d}$ are matched, then $\underline{h}=h_{d}$ for all $t$. Hence we can have an exact (not asymptotic) trajectory tracking if there are no modeling errors. The decoupling/partial linearization feedback law (10) can thus be called the nominal trajectory tracking control. But there still are joint and link vibrations in the nonlinear subsystem (12), so that a perturbed stabilization control acting as active damping of the undesirable elastic vibrations is required. Introducing this perturbation control eliminates the possibility of perfect tracking, but a fairly accurate tracking is still possible with a properly designed perturbed stabilization control.

\section{PERTURBED STABILIZATION CONTROL LAW SYNTHESIS}

In what follows, a combined linear quadratic stabilizer designed about the desired trajectory terminal state and robust servocompensator driven by the motor angle tracking error at each joint, is adopted as the perturbed stabilization control. For simplicity, we consider first-order compensators fed by the errors $\widetilde{\underline{h}}=\underline{h}-\underline{h}_{d}$. Thus the servocompensator is of the form

$$
\underline{\dot{x}}_{\mathrm{s}}=-\underline{\Lambda} \tilde{\tilde{h}}, \quad \underline{x}_{\mathrm{s}} \in \mathrm{R}^{\mathbf{n}}
$$

where $\Lambda$ is an $n \times n$ diagonal gain matrix. Define a composite system

$$
\underline{\hat{\mathrm{x}}}=\underline{\mathrm{F}} \underline{\hat{\mathrm{x}}}+\underline{\mathrm{G}} \delta \underline{v}
$$

where $\underline{\hat{x}}=\left(\delta \underline{z}^{\mathrm{T}}, \delta \underline{\eta}^{\mathrm{T}}, \underline{\mathrm{x}}_{\mathrm{S}}^{\mathrm{T}}\right)^{\mathrm{T}} ; \delta \underline{\mathrm{z}}$ and $\delta \underline{\eta}$ are the perturbations of $\underline{z}$ and $\eta$ respectively; and $\delta \underline{v}=-\underline{S} \underline{\hat{x}}$ can be obtained by minimizing a quadratic performance index $\mathrm{J}=\int_{0}^{\infty}\left(\underline{\hat{x}}^{\mathrm{T}} \underline{\mathrm{Q}}+\delta \underline{v}^{\mathrm{T}} \underline{\mathrm{R}} \delta \underline{v}\right) \mathrm{d}$ where $R$ is a symmetric positive definite matrix and $\vec{Q}$ is a symmetric positive semidefinite matrix. The final overall torques required to control the multi-link flexible arm can be computed by

$$
\underline{\tau}=\underline{\alpha}^{\prime}(\underline{x})+\underline{\beta}^{\prime}(\underline{x})(\underline{v} \mathbf{d}-\underline{S} \underline{\hat{x}})
$$

We further remark that to compensate for the tracking errors of the end effector caused by gravity, the quasi-static deflections must be taken into account in both the trajectory 
planning and LQ regulation. The overall control configuration is shown in Fig. 2.

\section{TWO-LINK FLEXIBLE MANIPULATOR EXAMPLE}

The proposed control strategy will be tested via computer simulation for a two-link manipulator as depicted in Fig. 3. The manipulator comprises links 1 and 2 with both joint and link flexibilities. The link coordinate systems attached to the undeformed and deformed links are also shown. The manipulator operates in the vertical plane and has the following specifications: length of each arm is $0.75 \mathrm{~m}$; payload mass (nominal) is $3.0 \mathrm{Kg}$; mass of joint 2 is $5.5 \mathrm{Kg}$; mass of link 1 is $2.2 \mathrm{Kg}$; mass of link 2 is $1.0 \mathrm{Kg}$; stiffness El of link 1 is $1220.0 \mathrm{~N}-\mathrm{m}^{2}$; stiffness EI of link 2 is $218.0 \mathrm{~N}-\mathrm{m}^{2}$; stiffness $\mathrm{k}_{1}$ of joint 1 is $15000 \mathrm{~N}-\mathrm{m} /$ rad; stiffness $k_{2}$ of joint 2 is $15000 \mathrm{~N}-\mathrm{m} / \mathrm{rad}$ : reflected moment of inertia $J_{\mathrm{r} 1}$ of joint 1 is $0.7916 \mathrm{Kg}-\mathrm{m}^{2}$; reflected moment of inertia $\mathrm{J}_{\mathrm{r} 2}$ of joint 2 is $0.5940 \mathrm{Kg}-\mathrm{m}^{2}$; moment of inertia $\mathrm{J}_{\mathrm{zz}}$ of payload is $0.0375 \mathrm{Kg}-\mathrm{m}^{2}$; and $\mathrm{n}_{1}=\mathrm{n}_{2}=1$. Two static deflection lines of a cantilever beam with only one concentrated force and only one concentrated torque acting on the free end of each link are selected as the vital shape functions [13]:

$$
\begin{aligned}
& \Phi_{\mathrm{i} 2}\left(\mathrm{x}_{\mathrm{i}}\right)=1.5\left[\left(\ell_{\mathrm{i}}+\mathrm{x}_{\mathrm{i}}\right) / \ell_{\mathrm{i}}\right]^{2}-0.5\left[\left(\ell_{\mathrm{i}}+\mathrm{x}_{\mathrm{i}}\right) / \ell_{\mathrm{i}}\right]^{3} ; \\
& \Phi_{\mathrm{i} 1}\left(\mathrm{x}_{\mathrm{i}}\right)=\left[\left(\ell_{\mathrm{i}}+\mathrm{x}_{\mathrm{i}}\right) / \ell_{\mathrm{i}}\right]^{2} \quad \mathrm{i}=1,2
\end{aligned}
$$

where $x_{i}$ is the coordinate along the axis of beam $i\left(x_{i}=-\ell_{i}\right.$ to 0$)$ and $\ell_{i}$ is the length of beam $i$. With the selected shape functions (18), the jk-th flexible stiffness $K_{\mathrm{jjk}}$ of ith link can be computed [20]: $\mathrm{K}_{111}=11567.407, \mathrm{~K}_{112}=\mathrm{K}_{121}=8675.555, \mathrm{~K}_{122}=$ 8675.555 , and $K_{211}=2066.962, K_{212}=K_{221}=1550.222, K_{222}=$ $1550.222 \mathrm{~N} / \mathrm{m}$.

For the present two-link case, $q=\left[q_{m 1}, q_{m 2}, q_{1}, q_{2}, q_{11}\right.$ $\left.\mathrm{q}_{12}, \mathrm{q}_{21}, \mathrm{q}_{22}\right]^{\mathrm{T}}$ and $\underline{x}=\left(\underline{x}_{1}^{\mathrm{T}}, \underline{x}_{2}^{\mathrm{T}}\right)^{\mathrm{T}}=\left(\mathrm{q}_{1}-\mathrm{q}_{\mathrm{m} 1}, \mathrm{q}_{2}-\mathrm{q}_{\mathrm{m} 2}\right.$, $q_{11}, q_{12}, q_{21}, q_{22}, q_{m 1}, q_{m 2}, \dot{q}_{1}-\dot{q}_{m 1}, \dot{q}_{2}-\dot{q}_{m 2}, \dot{q}_{11}, \dot{q}_{12}$, $\left.\dot{\mathrm{q}}_{21}, \dot{\mathrm{q}}_{22}, \dot{\mathrm{q}}_{\mathrm{m} 1}, \dot{\mathrm{q}}_{\mathrm{m} 2}\right)^{\mathrm{T}}$. In the nominal tracking control we set $\omega_{0}=4 \mathrm{rad} / \mathrm{sec}$. As for the perturbed stabilization control, we take $\Lambda_{2 \times 2}=10 \mathrm{I}, \mathrm{R}_{2 \times 2}=\mathrm{I}, \mathrm{Q}_{18 \times 18}=\operatorname{diag}\left[100 \mathrm{I}_{8 \times 8} 20 \mathrm{I}_{3 \times 3}\right.$ $\left.102010 I_{5 \times 5}\right]$ and use the ORACLS programs [22] to compute the feedback gain matrix $\underline{S}_{2 \times 18}$. In the simulation study, the arm is required to track the trajectory

$$
q_{1}(t)=q_{2}(t)=-\left[1 /\left(2 \pi^{2}\right)\right] \sin (2 \pi t)+(1 / \pi) t+\pi / 18
$$

for $0 \leqslant \mathrm{t} \leqslant 1$ sec. To compensate for the quasi-static deflections $\left(x_{f, 1}, x_{f, 2}, \ldots, x_{f, 6}\right)$ along the nominal trajectory, the commands required to generate the reference inputs are modified as follows:

$$
\begin{aligned}
x_{7, d}= & q_{1}(t)-x_{f, 1}-\left(x_{f, 3}+x_{f, 4}\right) / \ell_{1} \\
x_{8, d}= & q_{2}(t)-x_{f, 2}-\left(x_{f, 5}+x_{f, 6}\right) / \ell_{2} \\
& -\left[\Phi_{11}^{\prime}(0) x_{f, 3}+\Phi_{12}^{\prime}(0) x_{f, 4}\right]+\left(x_{f, 3}+x_{f, 4}\right) / \ell_{2}(20)
\end{aligned}
$$

for $0 \leqslant \mathrm{t} \leqslant 1 \mathrm{sec}$, where $\Phi_{\mathrm{ij}}^{\prime}(0)$ means the slope of $\Phi_{\mathrm{ij}}\left(\mathrm{x}_{\mathrm{i}}\right)$ at $\mathrm{x}_{\mathrm{i}}=$ 0 . Two sets of simulations are selected and shown in Figs. 4 and 5 , wherein $X 1$ and $X 2$ are the deflections of joint 1 and 2 , respectively; UE 1 and UE 2 are the tip deflections of link 1 and 2, respectively; $\mathrm{X} 7$ and $\mathrm{X} 8$ are the positions of motor 1 and 2 , respectively; XER and YER are, respectively, the $\mathrm{x}$ and $\mathrm{y}$ components of the task space trajectory error compared to the rigid arm counterpart with joint trajectory (19). In Fig. 4, only partial linearization control is used. The input-output pairs have perfect motor-based trajectory following, but the joint and link deflections are oscillatory. In Fig. 5, both partial linearization and perturbed stabilization control are applied. The undesirable joint and link oscillations are actively damped out by the LQR stabilizer. The perfect motor-based trajectory following is perturbed, thus the suggested control strategy has tradeoff between tracking and stabilization.

\section{CONCLUSION}

The effects of joint and link flexibility on the overall dynamic performance of a flexible robotic system are very significant. They are the sources of trajectory tracking errors and undesirable oscillations of the end effector. In this paper we have presented a design methodology based on the differential geometric structure algorithm and linear quadratic regulator theory. The proposed control strategy is tested via computer simulation for a two-link flexible arm with both joint and link flexibility. The simulation results indicate that large joint angle control and significant reduction in vibrations can be achieved. Even though the feedback control law is linear and simple, it does require the access to all the flexible states. Its experimental implementation deserves further investigation.

\section{ACKNOWLEDGMENT}

This research was supported by National Science Counci of R.O.C. under grant NSC 78-0422-E002-03.

\section{REFERENCES}

[1] O. Maizza-Neto, "Modal analysis and Control of Flexible Manipulator Arms," Ph.D. Thesis, Department of M. E. M. I. T., Cambridge, 1974.

[2] W. J. Book, O. Maizza-Neto, and D. E. Whitney, "Feedback Control of Two-Beam, Two-Joint Systems with Distributed Flexibility," ASME. J. Dynamic Syst., Meas., Control., vol. 97, pp. 424-431, 1975.

[3] E. Schmitz, "Dynamics and Control of a Planar Manipulator with Elastic Links," IEEE Proc. 25th Conf. Decision Contr., Athens, Greece, pp. 1135-1139, 1986.

[4] X. Ding, T. J. Tarn, and A. K. Bejczy, "A Novel Approach to the Modeling and Control of Flexible Robot Arms," IEEE Proc. 27th Conf. Decision Contr., Austin, Texas, pp. 52-57, 1986.

[5] J. Z. Sasiadek and R. Srinivasan, "Model Reference Adaptive Control for a Flexible Two-Link Manipulator Arm," Proc. IFAC Int. Symp. on the Theory of Robots, Vienna, Austria, Dec. 3-5, 1986.

[6] K. Oosting and S. L. Dickerson, "Simulation of a HighSpeed Lightweight Arm," IEEE Int. Conf. Robotics and Automation, Philadelphia, PA., pp. 494-496, 1988.

[7] E. Bayo, "Computed Torque for the Position Control of Open-Chain Flexible Robots," IEEE Int. Conf. Robotics and Automation, Philadelphia, PA., pp. 316-321, 1988.

[8] T. Komatsu, M. Uenohara, S. Iikura, H. Miura and I 
Shimoyama, "Active Vibration Control for Flexible Space Environment Use Manipulators," Dynamics of Controlled Mechanical Systems, edited by G. Schweitzer and $M$. Mansour, Springer-Verlag, Heidelberg, Germany, pp. 309-320, 1989.

[9] A. Das and S. N. Singh, "Dual Model Control of an Elastic Robotic Arm: Nonlinear Inversion and Stabilization by Pole Assignment," Proc. 1989 Amer. Contr. Conf., Pittsburgh, PA., pp. 1598-1603, 1989.

[10] J. Carusone, K. S. Buchan, and G. M. T. D'Eleuterio, "End-Effector Tracking Control for Structurally Flexible Manipulators," Proc. 1989 Amer. Contr. Conf., Pittsburgh, PA., pp. 1389-1396, 1989.

[11] P. J. Nathan and S. N. Singh, "Variable Structure Control of a Robotic Arm with Flexible Links," IEEE Int. Conf. Robotics and Automation, Scottsdale, AZ., pp. 882-887, 1989.

[12] T. Fukuda, "Flexibility Control of Elastic Robotic Arms," J. of Robotic Systems, Vol. 2, pp. 73-88, 1985.

[13] B. Gebler, "Feed-Forward Control Strategy for an Industrial Robot with Elastic Links and Joints," IEEE Int. Conf. Robotics and Automation, pp. 923-928, 1987.

[14] H. Henrichfreise, "The Control of an Elastic Manipulation Device Using DSP," Proc. 1988 Amer. Contr. Conf. Atlanta, Georgia, pp. 1029-1035, 1988.

[15] G. B. Yang and M. Donath, "Dynamic Model of a One-
Link Robot Manipulator with Both Structural and Joint Flexibility," IEEE Int. Conf. Robotics and Automation, Philadelphia, PA., pp. 476-481, 1988.

[16] G. B. Yang and M. Donath, "Dynamic Model of a TwoLink Robot Manipulator with Both Structural and Joint Flexibility," ASME 1988 Winter Annual Meeting, Chicago, Ill., pp. $37-44,1988$.

[17] A. Isidori, Nonlinear Control Systems: An Introduction, Lecture Notes in Control and Inf. Sci., Springer-Verlag, pp. 179-206, 1985.

[18] A. Isidori and J. W. Grizzle, "Fixed Modes and Nonlinear Noninteracting Control with Stability," IEEE Trans. on Automatic Control, vol. AC-33, pp. 907-914, 1988.

[19] L. C. Lin and K. Yuan, "Feedback Linearization and Linear Stabilization Control of Flexible Robots," Journal of Chinese Institute of Engineers, Vol. 13, pp. 11-24, 1990.

[20] L. C. Lin and K. Yuan, "A Lagrange-Euler-Assumed Modes Approach to Modeling Flexible Robotic Manipulators," Journal of Chinese Institute of Engineers, Vol. 11, pp. 335-347, 1988.

[21] F. F. Franklin, J. D. Powell, and A. Emami-Naeini, Feedback Control of Dynamic Systems, Addison-Wesley, Mass., 1986, pp. 336-341.

[22] E. S. Armstrong, ORACLS: A Design System for Linear Multivariable Control, New York: Marcell Dekker, 1980.

Fig. 3 Two-link flexible arm

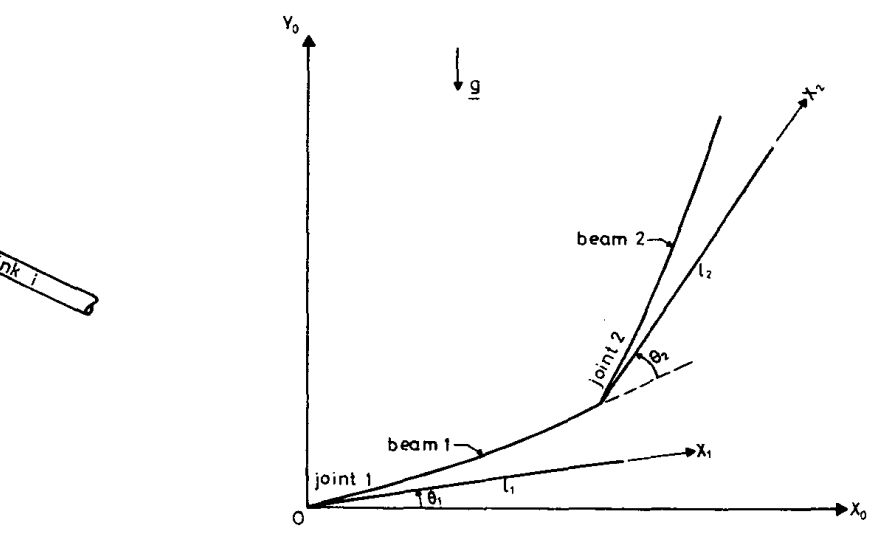

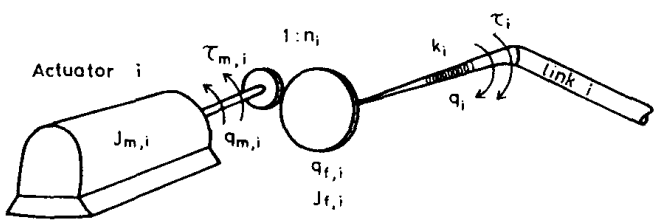

Fig. 1 Flexible joint model

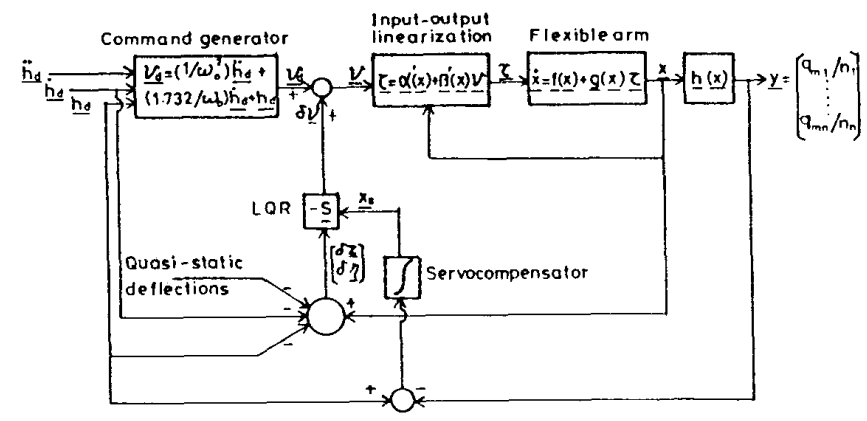

Fig. 2 Control configuration for flexible arm 


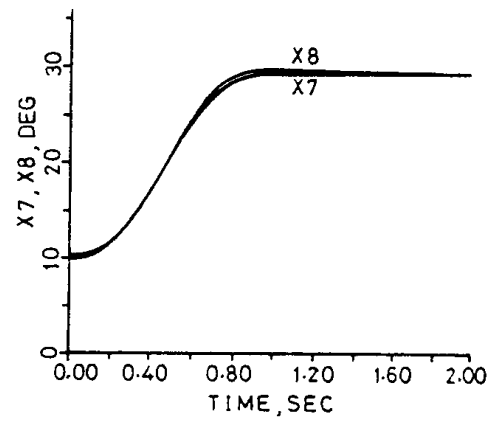

(a) Positions of motor 1, 2.

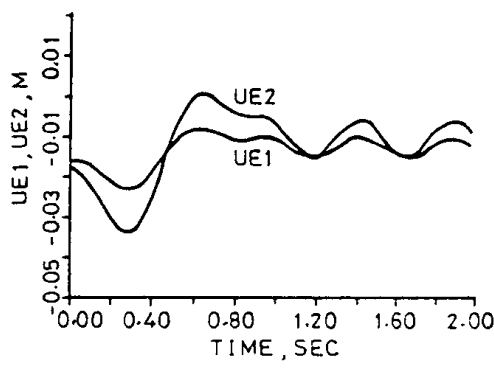

(b) Tip deflections of link 1, 2.

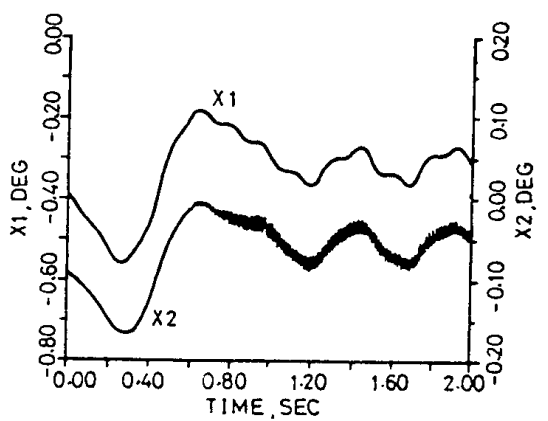

(c) Deflections of joint 1, 2.

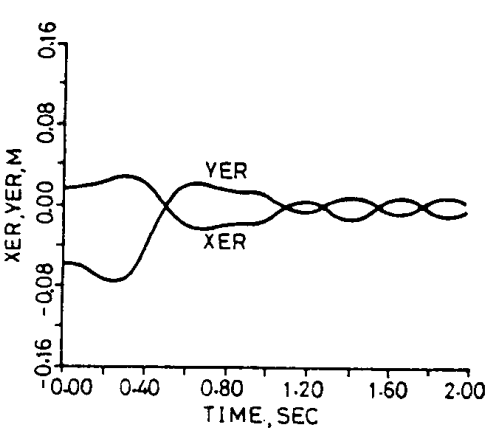

(d) Task space errors.

Fig. 4 Response curves with partial linearization only.

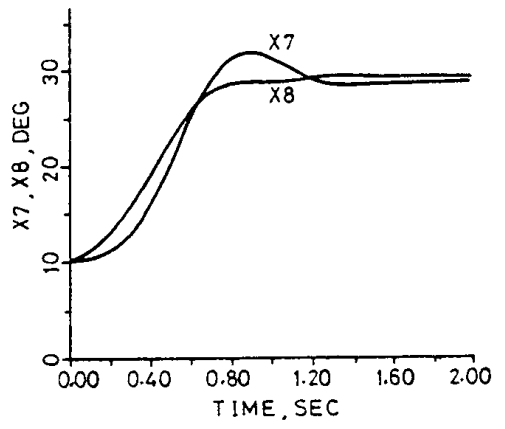

(a) Positions of motor 1,2 .

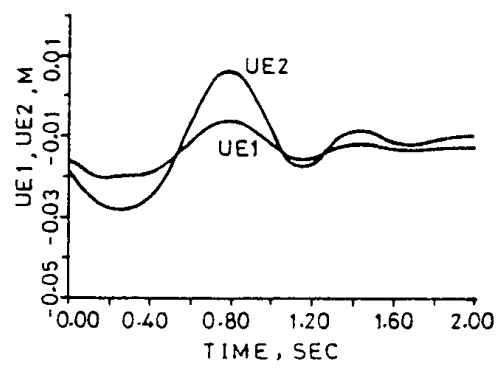

(b) Tip deflections of link 1, 2 .

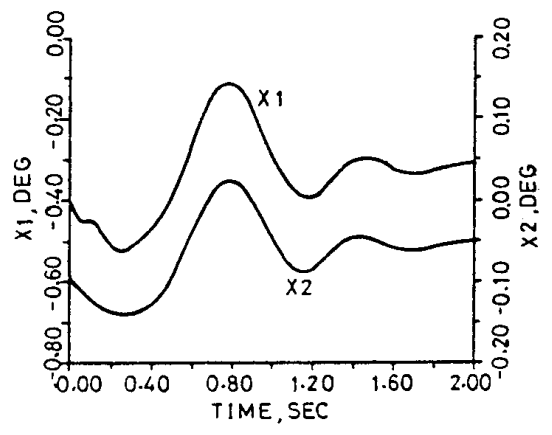

(c) Deflections of joint 1, 2 .

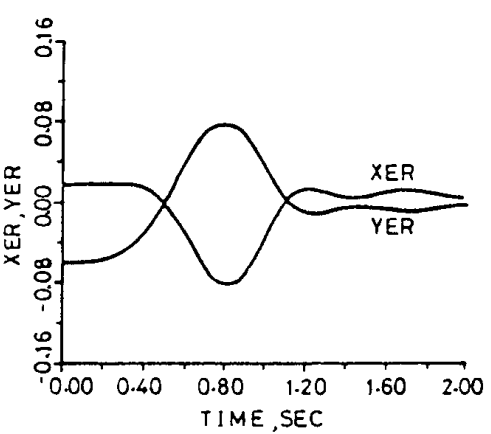

(d) Task space errors.

Fig. 5 Response curves with partial linearization and perturbed stabilization. 